\section{Reply to Petersen}

To the Editor-We appreciate Petersen's ${ }^{1}$ commentary on our study evaluating the use of rapid indicators in detecting residual debris in manually cleaned gastrointestinal endoscopes. ${ }^{2}$ He underscored the importance of several challenges related to the improvement of endoscope reprocessing, including the need for quality assurance. Failure to adhere to reprocessing guidelines occurs for various reasons, and protocol breaches have been associated with endoscope-related outbreaks. ${ }^{3}$ Methods for ensuring adherence to reprocessing protocols are lacking. Systematic culturing and rapid indicator testing have the potential to prevent pathogenic transmission by verifying process quality and identifying contamination prior to patient use.

More research is needed to fill knowledge gaps and develop workable solutions that ensure patient safety and are operationally feasible. Microbiological cultures may provide clinically relevant information, but as Petersen ${ }^{1}$ stated, there are several limitations associated with reliance on microbial cultures.

In addition to the points raised, the transmission of microorganisms may not always result in infection. Even if infection does ensue, it may not be immediately apparent after exposure to the endoscope because of silent transmission and colonization. ${ }^{4-6}$ In 1 recent endoscopy-associated, multidrug-resistant organism (MDRO) outbreak, investigators found that several patients were asymptomatic carriers of an extended-spectrum $\beta$-lactamase-producing Klebsiella pneumoniae for more than 10 months after exposure to contaminated endoscopes. Others presented with symptomatic bloodstream infections or biliary tract infections. Initial attempts to culture implicated duodenoscopes were unsuccessful, and it appeared that the endoscopes were free of viable microbes. More cases continued to occur, and investigators were able to harvest the epidemic strain and other MDROs (Morganella morganii and Pseudomonas aeruginosa) on several duodenoscopes, following a change in sampling and culturing protocols. ${ }^{4}$ This emphasizes the importance of microbial cultures but also highlights the potential disadvantages of relying solely on cultures, because they are difficult to perform and may not be a reliable indicator of the absence of viable organisms. In the outbreak previously mentioned, ${ }^{4}$ 253 patients were exposed to multiple MDROs over a period of 9 months, with 16 verified cases of transmission, because cultures failed to identify contaminated endoscopes. ${ }^{4}$ The lack of standardization in sampling and culturing techniques alluded to by Petersen ${ }^{1}$ may have played a role in the duration and severity of this outbreak.

Therefore, a reliable, rapid audit tool that can detect contaminated endoscopes before patient use would be valuable in verifying reprocessing effectiveness. As such, our study sought to determine the feasibility of using rapid indicators on clinically used endoscopes.

One limitation of this, our initial study, is the lack of terminal cultures following high-level disinfection to validate our methods of assessing contamination levels after bedside precleaning and manual cleaning. We recognize the importance of obtaining microbiological data after high-level disinfection and other reprocessing steps, but this was beyond the purview of our study. Further studies should use cultures to provide context to rapid indicators results. We have incorporated microbial cultures in our ongoing research to address this issue.

Petersen ${ }^{1}$ noted the water and surface adenosine triphosphate (ATP) tests and dipstick tests performed to detect blood and protein in channel effluent. Our team also employed protein tests on component surfaces (eg, distal ends, biopsy ports, and control handles). Even though cultures were not employed in this study, it should be noted that residual contamination within endoscope suction/biopsy channels after manual cleaning was assessed using previously microbiologically validated cutoffs for protein, hemoglobin, and ATP.,

Alfa et $\mathrm{al}^{7}$ established benchmarks for protein, hemoglobin, and bioburden to verify that clinically used endoscopes have been adequately manually cleaned. These benchmarks were used in a simulated-use study with artificial test soil to validate ATP levels in relative light units (RLUs). ${ }^{8}$ Researchers concluded that benchmarks for protein, hemoglobin, and bioburden (ie, less than $6.4 \mu \mathrm{g} / \mathrm{cm}^{2}, 2.2 \mu \mathrm{g} / \mathrm{cm}^{2}$, and $4 \log _{10}$ colony forming units $/ \mathrm{cm}^{2}$, respectively) were not exceeded when ATP measured less than 200 RLUs. ${ }^{8}$ Further research with clinically used endoscopes determined that protein and bioburden benchmarks for manual cleaning were met when ATP levels were less than 200 RLUs. ${ }^{9}$ Others have found a strong association between culture results and ATP levels. ${ }^{10-12}$ Alfa et $\mathrm{al}^{9}$ noted that ATP tests used to monitor cleaning effectiveness cannot reliably detect the small numbers of microbes that may be present in patient-ready endoscopes.

We used the benchmarks above to identify post-manual cleaning organic residue and bioburden that may reduce the efficacy of high-level disinfection. ${ }^{7,13}$ The tests detected potentially significant contamination that otherwise would not have been identified, given the currently recommended practice of visual inspection. As ongoing studies attempt to define optimal methods for assessing cleanliness, we maintain that rapid indicator tests could be used to detect persistent contamination that should be absent prior to high-level disinfection. As more endoscope-related outbreaks are reported, we must work to refine our understanding of endoscope reprocessing and contamination. Our findings suggest that the current practice of visual inspection is not adequate for assessing endoscope cleanliness. Rapid indicator testing may be 
useful while researchers continue to evaluate methods for reducing the risk of endoscope-related infection.

\section{ACKNOWLEDGMENTS}

We thank Evan Doyle, BS, for his editorial assistance.

Financial support. $3 \mathrm{M}$ provided funding and materials to Ofstead and Associates for the study discussed in B. Petersen's commentary (to which this letter replies). $3 \mathrm{M}$ did not have access to any study data and was not involved in the preparation of this letter. Additional research support was provided by Mayo Clinic and Ofstead and Associates. Neither the physicians at Mayo Clinic (K.H.V., P.K.T.) or the University of North Carolina School of Medicine (T.H.B) nor their departments received monetary compensation for participating in the study.

Potential conflicts of interest. C.L.O. reports that she is employed by Ofstead and Associates, which has received research funding and speaking honoraria related to infection prevention from $3 \mathrm{M}$, Johnson and Johnson, Ecolab, and Sanofi Pasteur. H.L.Y. and H.P.W. report that they are employed by Ofstead and Associates. All other authors report no conflicts of interest relevant to this article. All authors submitted the ICMJE Form for Disclosure of Potential Conflicts of Interest, and the conflicts that the editors consider relevant to this article are disclosed here.

Kavel H. Visrodia, MD; ${ }^{1}$ Cori L. Ofstead, MSPH; ${ }^{2,3}$ Hannah L. Yellin, BA; ${ }^{2}$ Harry P. Wetzler, MD, MSPH; ${ }^{2}$ Pritish K. Tosh, MD; ${ }^{3}$ Todd H. Baron, $\mathrm{MD}^{4}$

Affiliations: 1. Department of Internal Medicine, Mayo Clinic, Rochester, Minnesota; 2. Ofstead and Associates, Saint Paul, Minnesota; 3. Division of Infectious Diseases, Mayo Clinic, Rochester, Minnesota; 4. Division of Gastroenterology and Hepatology, University of North Carolina School of Medicine, Chapel Hill, North Carolina.

Address correspondence to Cori L. Ofstead, MSPH, Ofstead and Associates, 400 Selby Avenue, Suite V, Blair Arcade West, Saint Paul, MN 55102 (cori@ofsteadinsights.com).

Infect Control Hosp Epidemiol 2014;35(10):1309-1310

(C) 2014 by The Society for Healthcare Epidemiology of America. All rights reserved. 0899-823X/2014/3510-0017\$15.00. DOI: 10.1086/678075

\section{REFERENCES}

1. Petersen B. Monitoring of endoscope reprocessing: accumulating data but best practices remain undefined. Infect Control Hosp Epidemiol 2014;35:995-997.

2. Visrodia KH, Ofstead CL, Yellin HL, et al. The use of rapid indicators for the detection of organic residues on clinically used gastrointestinal endoscopes with and without visually apparent debris. Infect Control Hosp Epidemiol 2014;35:987-994.

3. Dirlam Langlay AM, Ofstead CL, Mueller NJ, et al. Reported gastrointestinal endoscope reprocessing lapses: the tip of the iceberg. Am J Infect Control 2013;41(12):1188-1194.

4. Aumeran C, Poincloux L, Souweine B, et al. Multidrug-resistant Klebsiella pneumoniae outbreak after endoscopic retrograde cholangiopancreatography. Endoscopy 2010;42(11):895-899.

5. Carbonne A, Thiolet JM, Fournier S, et al. Control of a multihospital outbreak of KPC-producing Klebsiella pneumoniae type 2 in France, September to October 2009. Euro Surveill 2010; 15(48):1-6.

6. Frias M, Tsai V, Moulton-Meissner $\mathrm{H}$, et al. Notes from the field: New Delhi metallo-beta-lactamase-producing Escherichia coli as- sociated with endoscopic retrograde cholangiopancreatography-Illinois, 2013. MMWR Morb Mortal Wkly Rep 2014;62(51/ 52):1051.

7. Alfa MJ, Degagne P, Olson N. Worst-case soiling levels for patient-used flexible endoscopes before and after cleaning. Am J Infect Control 1999;27(5):392-401.

8. Alfa MJ, Fatima I, Olson N. Validation of adenosine triphosphate to audit manual cleaning of flexible endoscope channels. Am J Infect Control 2013;41(3):245-248.

9. Alfa MJ, Fatima I, Olson N. The adenosine triphosphate test is a rapid and reliable audit tool to assess manual cleaning adequacy of flexible endoscope channels. Am J Infect Control 2013; 41(3):249-253.

10. Fushimi R, Takashina M, Yoshikawa $\mathrm{H}$, et al. Comparison of adenosine triphosphate, microbiological load, and residual protein as indicators for assessing the cleanliness of flexible gastrointestinal endoscopes. Am J Infect Control 2013;41(2):161-164.

11. Turner DE, Daugherity EK, Altier C, Maurer KJ. Efficacy and limitations of an ATP-based monitoring system. J Am Assoc Lab Anim Sci 2010;49(2):190-195.

12. Hansen D, Benner D, Hilgenhoner M, Leisebein T, Brauksiepe A, Popp W. ATP measurement as method to monitor the quality of reprocessing flexible endoscopes. Ger Med Sci 2004;2:Doc04.

13. Association for the Advancement of Medical Instrumentation (AAMI). Standard 58: Chemical Sterilization and High-Level Disinfection in Health Care Facilities. Arlington, VA: AAMI, 2010.

\section{Failure of a Hazard Analysis and Critical Control Point-Based Legionnaires' Disease Prevention Program: 2 Definite Nosocomial Cases Tell the Story}

To the Editor-In a recent article by Krageschmidt et al, ${ }^{1}$ it is surprising to read the authors' conclusion that the hazard analysis and critical control point (HACCP) method was an "effective" program for controlling Legionnaires' disease even though 2 confirmed nosocomial cases of Legionnaires' disease occurred during the study period while the HACCP approach was being followed. Irrespective of what number of cases might have been occurring before implementation of the HACCP approach (data not reported), the occurrence of 2 nosocomial cases in 2 years in the 2 study hospitals while the HACCP approach was used documents a failure to recognize source amplification and to stop subsequent disease transmission.

The inability to detect the etiologic source for 2 nosocomial cases raises questions about the validity of the environmental testing methods used to assess the overall success of the HACCP program. In particular, the environmental testing approach used by the authors may have been inadequately sensitive to detect the source of Legionella for these 2 cases. The testing methods as written in this study (only listed as previously described) are not detailed enough to determine 